\title{
Auto-eficacia Docente, Motivación del Profesor y Estrategias de Enseñanza
}

\author{
Susana Rodríguez ${ }^{1}$, José C. Núñez ${ }^{2}$, Antonio Valle ${ }^{1}$, \\ Rebeca Blas ${ }^{1}$, Pedro Rosario ${ }^{3}$ \\ ${ }^{1}$ Universidad de A Coruña, ${ }^{2}$ Universidad de Oviedo, ${ }^{3}$ Universidad de Minho, Portugal
}

Disponible online 22 diciembre de 2009

\begin{abstract}
Según los resultados de la investigación previa, los logros de los profesores en relación con sus actividades docentes van a depender, en buena medida, de la confianza que tengan en sí mismos para abordar todos estos cambios que conlleva su nuevo rol. En el presente estudio se han pretendido dos objetivos: a) aportar información sobre cómo los profesores coordinan diferentes ámbitos de autoeficacia (para optimizar el proceso instruccional, para gestionar el aula y para implicar al estudiante en el proceso de aprendizaje), y así dar lugar a perfiles homogéneos de autoeficacia como docentes; b) indagar acerca de cómo éstos se relacionan con niveles y tipos de motivación, estrategias instruccionales y con la autoestima de los docentes. En la investigación participaron 95 profesores de cinco universidades públicas españolas. Por una parte, en base al análisis de conglomerados se identificaron tres grupos de profesores con un perfil de autoeficacia docente distinto: 1) alta autoeficacia en las tres dimensiones, 2) media autoeficacia en optimización del proceso instruccional y en gestión del aula y medio-alta eficacia para la implicación del estudiante, y 3) baja autoeficacia en las tres dimensiones. Por otra parte, los resultados aportados por el ANOVA corroboran los hallazgos de investigaciones recientes en el sentido de que las creencias de auto-eficacia de los profesores tienen un papel crucial en el sostenimiento del compromiso con la enseñanza y en la motivación de los docentes.
\end{abstract}

Palabras Clave: auto-eficacia del profesor, motivación, autoestima, estrategias instruccionales

According to previous research, teachers' efficacy relating to teaching practices is highly dependent on the extent to which they are confident about their own capabilities to manage the new demands on their professional role. The present work aimed at: a) gathering information about the way teachers manage different aspects of self-efficacy (i.e., enhancing their instructional process in order to optimize their lessons and engage the students in the learning process), in order to identify homogeneous self-efficacy profiles, b) investigating the way these profiles relate to different levels and types of motivation, teaching strategies and teachers' self-esteem. Ninety-five teachers from five Spanish public Universities participated in this study. Three different profiles of teachers' efficacy have been identified: 1) high self-efficacy in the three dimensions; 2) medium self-efficacy in the enhancement of the teaching process and of lessons management, and medium-high efficacy in the engagement of students, and 3) low self-efficacy in the three dimensions. ANOVA results supported previous findings since teachers' self-efficacy beliefs played a crucial role on teachers' motivation and professional engagement.

Keywords: teacher's self-efficacy, motivation, self-esteem, teaching strategies

Este trabajo forma parte de otras investigaciones más amplias que han sido financiadas por la DGI-MEC (SEJ2006-01518) y por la DGU-MEC (EA2007-0242).

Correspondencia: Susana Rodríguez. Departamento de Psicoloxía Evolutiva e da Educación, Universidad de A Coruña, Campus de Elviña, s/n. E-mail: susana@udc.es. 
El docente universitario "se enfrenta hoy a una situación de cambio en la que se le solicita que asuma un rol diferente al hasta ahora asignado" (Rodríguez, 2002, pp. 53); no solamente debe ser un conocedor de la ciencia, un experto en técnicas y un investigador, sino que debe ser guía y supervisor de la formación científica del estudiante. La cuestión central radica en la necesidad de reconversión del rol tradicional de los profesores que de su clásico papel de transmisores del conocimiento deben llegar al papel de organizador y guía del aprendizaje, facilitador que estructura situaciones, motiva, orienta sobre procesos, presenta información básica, sugiere fuentes de datos, y tutela y evalúa realizaciones (Álvarez y García, 2002; González y Wagenaar, 2003). La tutoría universitaria se convierte así en un elemento dinamizador del proceso formativo académico, personal y profesional del alumnado, orientado de forma individualizada para crear alumnos emprendedores que sepan y puedan generar su propio trabajo como profesionales autónomos.

Uno de los grandes retos a los que se enfrentan los profesores universitarios consiste en adaptar sus estrategias de enseñanza al nuevo modelo que caracteriza la educación universitaria. Desde esta perspectiva, los logros de los profesores en relación con sus actividades docentes van a depender, en buena medida, de la confianza que tenga en sí mismos para abordar todos estos cambios que conlleva su nuevo rol. Según los resultados derivados de la investigación realizada sobre este tema, los sentimientos de competencia y de eficacia personal no sólo parecen influir en las expectativas de éxito como docentes, sino que tienen claras consecuencias sobre la motivación y el rendimiento de sus estudiantes (Skaalvik y Skaalvik, 2007; Wolters y Daugherty, 2007). A este respecto, enseñar a los profesores a ser más auto-eficaces debería contribuir a que los alumnos mejoren sus capacidades para gestionar los recursos personales y contextuales vinculados a un aprendizaje de calidad y, como consecuencia, mejoren también su grado de motivación y sus resultados académicos.

Los expertos consideran que la auto-eficacia docente engloba todo un conjunto de creencias que tiene el profesor sobre su propia capacidad para ejercer un efecto positivo en el aprendizaje de los alumnos. Tschannen-Moran y Woolfoolk (2001) afirman que aquellos profesores con altas creencias de auto-eficacia presentan mayor apertura a nuevas ideas, mayor disposición para probar nuevos métodos, mejor planificación y organización de sus clases y se muestran más entusiastas en el proceso de enseñanza. Además, la auto-eficacia docente no sólo se relaciona con el rendimiento de los alumnos sino también con la motivación que los docentes manifiestan en el aula y con las expectativas que tienen sobre sus estudiantes (Bamburg, 2004).

Los profesores que confían en su capacidad para enseñar y creen que una enseñanza eficaz influye en el aprendizaje de los alumnos, tienen mayor persistencia y se centran más en los aspectos académicos de su actividad que aquellos profesores con bajas expectativas respecto a su capacidad para influir en el aprendizaje de los alumnos (Gibson y Dembo, 1984). Según estos autores, aquellos profesores con alta auto-eficacia emplean más tiempo en la clase para el desarrollo de actividades académicas, utilizan métodos instructivos más complejos, proporcionan más ayuda y orientación a los alumnos y elogian sus logros académicos (Skaalvik y Skaalvik, 2007; Wolters y Daugherty, 2007). Por el contrario, los profesores con baja auto-eficacia dedican más tiempo de clase a tareas no académicas, retiran el apoyo a los alumnos cuando no alcanzan relativamente pronto los resultados previstos y critican los errores que éstos cometen.

Otros trabajos demuestran que la auto-eficacia del profesor se relaciona positivamente con el rendimiento de los alumnos, con la motivación, con el desarrollo de técnicas instructivas innovadoras y con el establecimiento de un clima positivo dentro del aula (Hoy y Woolfolk, 1990). Además, los profesores con mayor auto-eficacia confían más en los alumnos y, por tanto, son más capaces de compartir con ellos la responsabilidad a la hora de resolver los problemas que pueden surgir en el aula y muestran una mayor satisfacción con su trabajo como docentes (Klassen, et al., 2009).

A la luz de las citadas investigaciones que destacan el decisivo impacto del nivel de auto-eficacia docente sobre el desempeño de las funciones propias de su rol y, como consecuencia, la mejora del aprendizaje y rendimiento de los alumnos, en esta investigación se pretenden identificar posibles perfiles de auto-eficacia que presentan los docentes universitarios y analizar sus diferencias en cuanto a motivación y a las estrategias de enseñanza que utilizan. En consecuencia con esta nueva perspectiva, en la presente investigación no se realizan predicciones concretas debido al carácter predominantemente exploratorio del estudio. Al igual que se está abordando el constructo de metas académicas desde una perspectiva del múltiples metas (p.e., Pintrich, 2000; Valle, Cabanach, Núñez, González-Pienda, Rodríguez y Piñeiro, 2003; Valle, Núñez, Cabanach, Rodríguez, González-Pienda y Rosário, 2009; Valle, Núñez, Rodríguez, Cabanach, González-Pienda y Rosário, en prensa), se intentarán identificar grupos de profesores diferentes sobre la base de su perfil de auto-eficacia docente, constituido por la combinación de diferentes niveles de tres dimensiones más específicas de auto-eficacia (eficacia percibida para optimizar el proceso instruccional, eficacia percibida para gestionar el aula y eficacia percibida para implicar al estudiante en el proceso de aprendizaje). Una vez identificados estos perfiles, se investigará cómo se relacionan con niveles y tipos motivacionales, estrategias instruccionales y con la autoestima. 
Método

\section{Participantes}

La muestra final estaba integrada por 95 profesores (58.90\% hombres y $41.10 \%$ mujeres) pertenecientes a dieciséis titulaciones de cinco universidades públicas españolas. Estos profesores, pertenecientes a diez centros universitarios distintos, impartían docencia en las siguientes titulaciones: Enfermería, Químicas, Podología, Empresariales, Psicología, Periodismo, Comunicación Audiovisual, Logopedia, Fisioterapia, Psicopedagogía, Maestro en Educación Física, Maestro en Audición y Lenguaje, Maestro en Educación Especial, Maestro en Lengua Extranjera y Maestro en Educación Infantil. De ellos, el 66.30\% imparte docencia en primer ciclo y el $33.70 \%$ en segundo ciclo. En cuanto a la distribución por edades, el $21.10 \%$ tienen menos de 40 años, el $35.70 \%$ entre 40 y 50 años, el $33.70 \%$ entre 50 y 60 años y el $9.50 \%$ tienen más de 60 años. Los participantes se implicaron de modo voluntario en la investigación.

\section{Instrumentos}

Escala de Auto-eficacia Docente. Para evaluar la autoeficacia docente se utilizó una versión adaptada al español de la Teachers' Sense of Efficacy Scale de Tschannen-Moran y Woolfolk (2001). A través de esta escala se mide la percepción de auto-eficacia docente en tres dimensiones: (a) eficacia percibida para optimizar la propia instrucción, (b) eficacia percibida para gestionar el aula y (c) eficacia para implicar al estudiante en el aprendizaje. Esta escala se ha mostrado muy fiable y con excelente validez (Klassen et al., 2009). La fiabilidad de la escala total es elevada $(\alpha=.92)$, siendo también excelentes la fiabilidad de las tres subescalas [eficacia percibida para optimizar la propia instrucción $(\alpha=.81)$, eficacia percibida para gestionar el aula $(\alpha=.86)$, eficacia para implicar al estudiante en el aprendizaje $(\alpha=.81)]$.

Autoestima. Los docentes respondieron a un conjunto de 10 ítems referidos a su autoestima como docente (p.e., "En general, creo que soy un buen profesor"). Los ítems de esta escala han sido elaborados para esta investigación siguiendo el modelo de autoestima de Rosenberg. La consistencia interna de esta escala es satisfactoria $(\alpha=.90)$.

Cuestionario de Motivación Docente. Siguiendo la Teoría de Metas (Ames, 1994; Brophy, 2004; Dweck y Leggett, 1988, Elliot y McGregor, 2001; Pintrich, 2003) se ha elaborado un cuestionario en el que cada profesor valoró en qué medida diferentes razones explicaban su implicación en la actividad docente. El análisis factorial permitió diferenciar tres dimensiones motivacionales: a) orientación motivacional intrínseca, b) motivación centrada en el yo, c) desmotivación. Por tanto, se diferenció entre razones intrínsecas para preparar las clases y los materiales docentes (p.e., "me esfuerzo en preparar las clases o los materiales porque me gusta ver como mis alumnos aprenden"), razones para implicarse en el trabajo docente más vinculadas a la imagen como profesor (p.e., "me esfuerzo en preparar las clases o los materiales porque es importante para mi que mis compañeros me consideren un gran profesor/a”), así como tendencias docentes desmotivadoras del tipo: "da igual que te esfuerces más o menos en la enseñanza, recibes el mismo pago" y que pueden entenderse como razones explicativas de la falta de implicación con la actividad docente. Los tres factores obtenidos explican conjuntamente el $77.9 \%$ de la varianza total. La fiabilidad de las escalas es aceptable: motivación intrínseca, $\alpha=.76$; desmotivación, $\alpha=.78$ y motivación hacia el yo, $\alpha=.88$.

Cuestionario de Estrategias Instruccionales. Siguiendo los planteamientos del modelo sociocognitivo del aprendizaje autorregulado (Zimmerman, 1998, 2000), se ha construido un cuestionario con el fin de valorar las tres fases propias de una enseñanza autorregulada: "antes" (nivel de implicación en la planificación del proceso instruccional, "durante" (implementación de actividades) y "después” (evaluación de logros e implicaciones). No obstante, mediante el análisis factorial se diferenciaron únicamente dos factores: a) un factor que evalúa el nivel en el que docente planifica su actividad instruccional (p.e., "estructuro de forma lógica la información que doy en clase”) y, b) otro que aporta información en torno a la medida en que el profesor desarrolla diferentes actividades para supervisar su práctica instruccional en el aula (p.e., "intento constantemente demostrar a los alumnos los progresos que van realizando"), aglutinando las fases de implementación y evaluación. La varianza conjunta explicada por estos dos factores, de planificación y supervisión de la enseñanza, alcanzó el $63 \%$, lo cual indica que esta es una estructura factorial consistente, así como también lo es la propia consistencia interna de cada uno de los factores (planificación, $\alpha=.80$, supervisión, $\alpha=.78$ ).

\section{Procedimiento}

Los datos relativos a las variables estudiadas fueron recogidos en cada uno de los centros universitarios de los que formaban parte los profesores que participaron en la investigación. Los cuestionarios fueron aplicados en un único momento temporal por personal especializado que colaboró en la investigación. A los participantes, que contestaron de forma individual y sin límite de tiempo a cada uno de los cuestionarios, se les recordaba que era muy importante que respondieran sinceramente a las distintas cuestiones planteadas.

\section{Análisis de datos}

Con el fin de conocer el perfil de auto-eficacia docente de 
los profesores que participaron en la investigación se recurrió al análisis de conglomerados para establecer los distintos grupos en función de los niveles que presentaban en cada una de las dimensiones. El objetivo fundamental de este tipo de análisis es el de identificar grupos de individuos sin que a priori se conozca criterio de agrupamiento alguno, de modo que se cumplan dos hechos importantes: que cada grupo sea lo más homogéneo posible y que sean lo más heterogéneos entre sí (Catena, Ramos y Trujillo, 2003). Dicho análisis se realizó mediante el procedimiento de agrupamiento K-medias, el cual es un método de partición que implica, al contrario que los métodos jerárquicos, que durante todo el proceso un sujeto puede asignarse a un grupo determinado y en pasos sucesivos reasignarse a cualquier otro cluster. La elección del número de conglomerados finales se llevó a término utilizando criterios tanto teóricos como estadísticos. Teóricamente se exigió que hubiera lógica en los perfiles con el conocimiento actual que se dispone de las dimensiones de la auto-eficacia. A nivel estadístico, por una parte, se exigió que el número de iteraciones necesarias para la convergencia no fuera mayor de diez y, por otra parte, que los perfiles fueran significativamente heterogéneos (para lo cual ha sido necesario realizar los correspondientes análisis de varianza).

Después de haber definido los perfiles de auto-eficacia docente a través del análisis de conglomerados, se intentó comprobar si había diferencias estadísticamente significativas entre los grupos en la motivación del profesor, en las estrategias de enseñanza y en la autoestima como docente. Para conocer las posibles diferencias entre los grupos se recurrió al análisis de varianza de un factor (ANOVA).

$$
\text { Resultados }
$$

\section{Perfiles de auto-eficacia docente}

Los grupos de auto-eficacia se han definido a partir de las diferentes combinaciones de las tres dimensiones que mide la Escala de Auto-eficacia Docente. Para eliminar el efecto debido a las diferencias en la medida de las tres subescalas de auto-eficacia (ya que el número de ítems de cada una de las subescalas no es el mismo), se realizó el análisis de conglomerados después de haber transformado las puntuaciones directas en típicas (en este caso, puntuaciones $z$ ).

Como se ha indicado anteriormente, para decidir el número de conglomerados se siguió el criterio habitual de tomar como válidas aquellas soluciones que convergen antes de las 10 iteraciones predeterminadas. Teniendo en cuenta que sólo las soluciones de dos y tres conglomerados cumplían con ese requisito, se optó por esta última ya que era la que ofrecía una diferenciación entre los grupos más acorde con las aportaciones teóricas y empíricas sobre el tema. En la Tabla 1 se presentan las medias y desviaciones típicas de las tres dimensiones de auto-eficacia en los tres perfiles.

Tabla 1. Estadísticos descriptivos de las dimensiones de auto-eficacia docente para cada uno de los tres perfiles.

\begin{tabular}{lccc}
\hline Dimensiones de auto-eficacia y perfiles & $\boldsymbol{N}$ & $\boldsymbol{M}$ & $\boldsymbol{D T}$ \\
Eficacia para lograr la implicación del estudiante & & \\
$\quad$ Perfil de baja auto-eficacia & 27 & 3.13 & .28 \\
Perfil de auto-eficacia media & 26 & 3.51 & .29 \\
Perfil de alta auto-eficacia & 42 & 4.05 & .26 \\
Eficacia para el manejo de estrategias instruccionales & & \\
Perfil de baja auto-eficacia & 27 & 3.26 & .30 \\
Perfil de auto-eficacia media & 26 & 3.64 & .22 \\
Perfil de alta auto-eficacia & 42 & 4.28 & .26 \\
Eficacia para el manejo de la clase & & & \\
Perfil de baja auto-eficacia & 27 & 3.13 & .39 \\
Perfil de auto-eficacia media & 26 & 4.02 & .32 \\
Perfil de alta auto-eficacia & 42 & 4.25 & .33 \\
\hline
\end{tabular}

Con el fin de ver si los tres grupos eran significativamente diferentes entre sí, como ya se mencionó, se realizó un ANOVA en el que se observó que existían diferencias estadísticamente significativas entre los tres grupos de profesores en las tres dimensiones de la auto-eficacia que configuran los perfiles: eficacia para lograr la implicación del estudiante $[F(2,92)=96.44 ; p=.000]$, eficacia para utilizar estrategias instruccionales $[F(2,92)=130.12 ; p=.000]$ y eficacia para el manejo de la clase $[F(2,92)=10.74 ; p=.000]$. Las comparaciones múltiples han resultado estadísticamente significativas en todos los casos posibles (incluso para los grupos de auto-eficacia media y baja en la dimensión de eficacia para el manejo de clase, en donde la diferencia de medias era menor que el resto de casos).

En suma, tanto si atendemos al criterio estadístico como al de la significación teórica y psicológica, la solución de tres grupos de perfiles parece muy razonable y, por ello, es la que se ha tomado en consideración en este trabajo.

En la línea de caracterizar a los tres grupos obtenidos, indicar que los resultados del análisis de conglomerados (ver figura 1) permiten identificar tres grupos ampliamente diferenciados por los niveles de auto-eficacia docente en cada una de las tres dimensiones evaluadas. Así, el grupo $1(n=$ 27) se caracteriza por unos niveles bajos de auto-eficacia docente en las tres dimensiones evaluadas (eficacia para lograr la implicación del estudiante, eficacia para utilizar estrategias instruccionales y eficacia para el manejo de la clase). 
Figura 1. Representación gráfica de los grupos de auto-eficacia docente identificados a través del análisis de conglomerados.

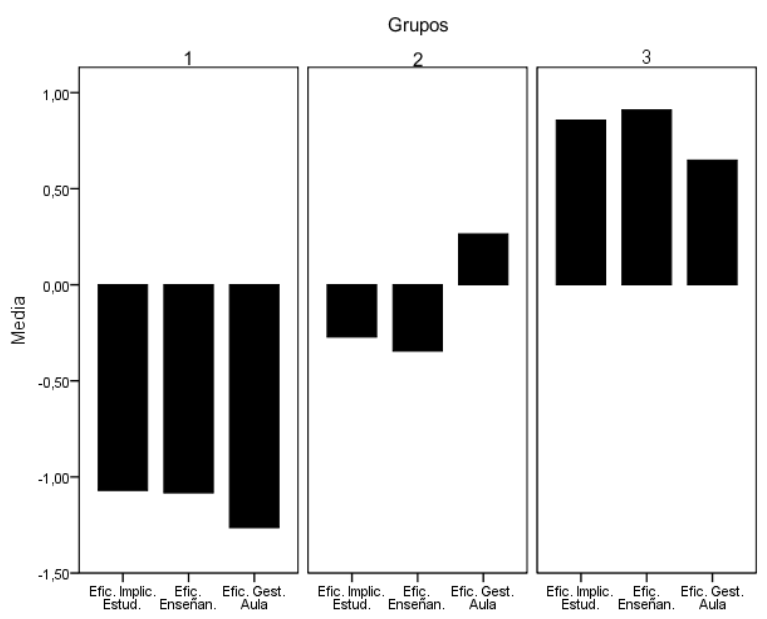

Efic. Implic. Estud.: eficacia para lograr la implicación del estudiante; Efic. Enseñan.: eficacia para utilizar estrategias instruccionales; Efic. Gest. Aula: eficacia para gestionar el aula.

El grupo $2(n=26)$ se caracteriza por una eficacia intermedia - aunque por debajo de la media - a la hora de implicar al alumno en el propio aprendizaje y para mejorar su propia docencia, y niveles también intermedios - pero por encima de la media - en la auto-eficacia percibida para gestionar el aula.

Finalmente, el grupo $3(n=42)$ se caracteriza por unos niveles altos de auto-eficacia docente en las tres dimensiones evaluadas.

De este modo, el grupo más numeroso de docentes encuestados $(44.20 \%)$ podrían caracterizarse por una alta percepción de eficacia en los tres aspectos de la actividad docente contemplados. Otro número importante de docentes $(27.40 \%)$ muestran niveles de auto-eficacia intermedios por debajo de la media para implicar al alumno en el aprendizaje y para mejorar sus actividades instruccionales, y unos niveles de auto-eficacia intermedios superiores a la media para gestionar el aula. Por último, el $28.40 \%$ de la muestra de profesores universitarios encuestados se caracterizan por su baja auto-eficacia percibida.

Perfiles de auto-eficacia docente, motivación y estrategias de enseñanza

Tal y como podemos observar en la Tabla 2 las diferencias entre los grupos son significativas para todas las variables contempladas, excepto para la motivación centrada en el yo. El grupo 1 evidencia los niveles significativamente más bajos de motivación intrínseca de los tres conglomerados y agrupa a docentes más desmotivados que los que integran el grupo de mayor auto-eficacia percibida (cluster 3). Según estos resultados, no parecen existir diferencias significativas en desmotivación entre los grupos 1 y 2 .
Las diferencias en autoestima son significativas entre los grupos y nos permiten asociar al conglomerado de profesores que se perciben más eficaces con los niveles más altos de autoestima, seguido del grupo con una confianza intermedia en su labor docente. El grupo de profesores con más baja percepción de eficacia son también el grupo de docentes con más baja autoestima de los tres. En síntesis, las tendencias observadas parecen corroborar que los docentes con más baja percepción de eficacia evidencian una baja autoestima y sostienen pocas razones intrínsecas para implicarse en la enseñanza, mientras que los docentes con niveles más altos de auto-eficacia se implican, en mayor medida, en la enseñanza por razones intrínsecas. Sin embargo, los niveles intermedios de auto-eficacia podrían asociarse también a creencias desmotivadoras en torno a la función educativa, al tiempo que los docentes con más confianza en su habilidad profesional pueden mostrar los más altos índices de autoestima y ser los docentes que tienen una más baja desmotivación.

Tabla 2. Medias, desviaciones típicas y significación estadística de las diferencias entre los perfiles de auto-eficacia docente respecto a la motivación del profesor y a las estrategias de enseñanza.

\begin{tabular}{|c|c|c|c|c|c|c|c|}
\hline & \multicolumn{2}{|c|}{$\begin{array}{l}\text { Grupo } 1 \\
{[n=27]}\end{array}$} & \multicolumn{2}{|c|}{$\begin{array}{c}\text { Grupo } 2 \\
{[n=26]}\end{array}$} & \multicolumn{2}{|c|}{$\begin{array}{l}\text { Grupo } 3 \\
{[n=42]}\end{array}$} & \multirow[t]{2}{*}{$F$} \\
\hline & $M$ & $D T$ & $M$ & $D T$ & $M$ & $D T$ & \\
\hline $\begin{array}{l}\text { Motivación centrada en } \\
\text { el yo }\end{array}$ & 3.17 & 0.69 & 2.85 & 0.99 & 2.59 & 1.15 & 2.72 \\
\hline Motivación intrínseca & 3.72 & 0.52 & 4.44 & 0.68 & 4.48 & 0.51 & $16.88 * * *$ \\
\hline Desmotivación & 2.76 & 0.96 & 2.56 & 1.24 & 2.12 & 0.97 & $3.28 *$ \\
\hline Autoestima & 3.17 & 0.48 & 3.87 & 0.28 & 4.21 & 0.34 & $62.67 * * *$ \\
\hline $\begin{array}{l}\text { Planificación de la ense- } \\
\text { ñanza }\end{array}$ & 3.28 & 0.46 & 4.18 & 0.47 & 4.48 & 0.38 & $64.55^{* * *}$ \\
\hline Supervisión de la enseñanza & 2.78 & 0.63 & 3.08 & 0.73 & 3.69 & 0.61 & $17.49 * * *$ \\
\hline
\end{tabular}

$* \mathrm{p}<.05, * * \mathrm{p}<.01, * * * \mathrm{p}<.001$

En el análisis de las relaciones entre la percepción de eficacia y practica docente hay que indicar que los docentes caracterizados por una alta auto-eficacia en los tres ámbitos contemplados (cluster 3) evidencian las medias significativamente más altas de los tres grupos, tanto en la planificación como en la supervisión de su labor docente. El grupo 2 caracterizado por una auto-eficacia media se diferencia del grupo que reúne a los docentes con más baja percepción de eficacia en la preparación de la docencia, pero no en las estrategias que efectivamente implementa en su actividad de aula. De este modo, una confianza intermedia en las posibilidades de mantener la disciplina y las normas del aula, para motivar al alumno o para mejorar la propia docencia podría no ser suficiente para mejorar la actuación estratégica del docente en el aula. 
Figura 2. Valores medios de cada uno de los grupos de auto-eficacia docente en las variables referidas a la motivación del profesor y a las estrategias de enseñanza.

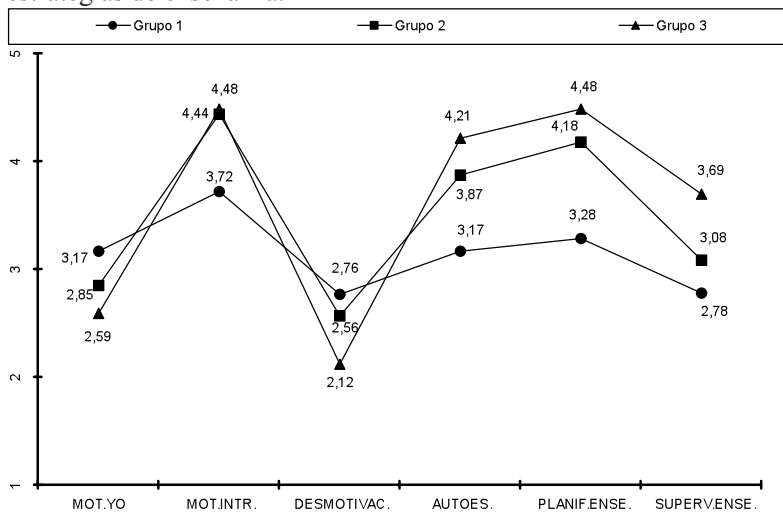

MOT.YO = Motivación centrada en el yo, MOT.INTR. = Motivación intrínseca, DESMOTIV.$=$ Desmotivación, AUTOES. $=$ Autoestima del profesor, PLANIF.ENSE. = Planificación de la enseñanza, SUPERV. ENSE. $=$ Supervisión de la enseñanza

\section{Discusión}

En este trabajo se pone de manifiesto que la muestra de profesores participantes puede diferenciarse en función de sus niveles de auto-eficacia percibida para implicar al alumnado en el aprendizaje, para optimizar la propia actividad docente y para gestionar el aula (Tschannen-Moran y Woolfolk, 2001). Además, los resultados encontrados permiten sugerir que en torno al 30\% del profesorado que participó en el estudio puede caracterizarse por una baja percepción de auto-eficacia en estas tres dimensiones.

Por otra parte, los resultados obtenidos corroboran los hallazgos de investigaciones recientes en el sentido de que las creencias de auto-eficacia de los profesores tienen un papel crucial en el sostenimiento del compromiso con la enseñanza y en la motivación de los docentes (Bamburg, 2004; Caprara, Barbaranelli, Borgogni, Petitta y Rubinacci, 2003; Caprara, Barbaranelli, Borgogni, y Steca, 2003). De hecho, las razones intrínsecas como el sentido del deber o el disfrute con el aprendizaje del alumno pueden ser significativamente más importantes entre los profesores caracterizados por una percepción de eficacia alta que entre los profesores con niveles intermedios y bajos de auto-eficacia. Al mismo tiempo, la falta de motivación y la desilusión con el trabajo se perciben como más evidentes entre los profesores que no se consideran eficaces para motivar al alumno, para mejorar la propia actividad docente y para controlar el aula. La desmotivación y la desilusión con la labor educativa pueden llegar a ser importantes incluso entre aquellos docentes que están sosteniendo niveles intermedios de competencia profesional. De hecho, se han encontrado diferencias significativas en esta variable entre el grupo de profesores con niveles intermedios de auto-eficacia y aquellos caracterizados por altos niveles de auto-eficacia.
Del mismo modo, observamos que el nivel de auto-eficacia docente puede asociarse con la autoestima del profesor. Si bien es cierto que no existen relaciones directas entre la autoestima y la auto-eficacia, ya que percibir una baja auto-eficacia docente puede no afectar a los niveles de autoestima personal (Woolfok, 2004), los profesores con altas creencias de auto-eficacia para implicar a los alumnos, mejorar su labor y gestionar el aula pueden ser individuos más satisfechos con ellos mismos, más seguros y más capaces de dirigir sus propias vidas. Estas valoraciones respecto a uno mismo pueden ser menos positivas entre los profesores que confían menos en su propia eficacia docente.

Finalmente, en consonancia con los trabajos que han vinculado la auto-eficacia percibida con la calidad de la planificación y control de la actividad docente (Allinder, 1994; Cousins y Walker, 1995a, 1995b; Guskey, 1988; Stein y Wang, 1988), nuestros resultados sugieren que la preparación de las clases, la mejora periódica de los contenidos y la formación continuada son más importantes para los profesores con alta percepción de eficacia. A este respecto, cabe indicar que si bien la preparación de la actividad instruccional puede ser más relevante entre los profesores con niveles altos y medios de auto-eficacia percibida, la supervisión en el aula de esa actividad instruccional es más efectiva entre el grupo de profesores con las tasas más altas de auto-eficacia percibida que entre los docentes con niveles medios o bajos. Posiblemente, la evaluación continua en el aula del progreso del alumno y el constante ajuste de las secuencias instruccionales a las necesidades del alumnado requiere de un alto grado de confianza en las propias habilidades docentes.

En síntesis, los resultados de este trabajo parecen confirmar que los docentes con baja percepción de eficacia evidencian una baja autoestima y no suelen aludir a razones intrínsecas para implicarse en la enseñanza mientras que los docentes con niveles más altos de auto-eficacia se implicarían en mayor medida en la enseñanza por razones intrínsecas, pudiendo mostrar más altos niveles de autoestima y ser los docentes que tienen una mayor motivación para la enseñanza.

Probablemente, la confianza en las posibilidades de controlar los comportamientos disruptivos y para hacer cumplir las normas puede contribuir a que el profesor dedique esfuerzos a la preparación y mejora de la docencia que imparte. Al mismo tiempo, percibirse capaz de conseguir que los alumnos valoren, comprendan y sean críticos con los contenidos que aprenden y percibirse eficaz en el uso de diferentes estrategias instruccionales puede hacer que el profesor se muestre más cómodo en el aula, que facilite más feedback informativo a sus alumnos, se arriesgue a innovar y, en general, construya en el aula un clima más participativo.

\section{Referencias}

Allinder, R. (1994). The relationship between efficacy and the instructional practices of special education teachers and consultants. Teacher Education and Special Education, 17, 86-95. 
Álvarez, V. y García, E. (2002). Orientación del aprendizaje en la enseñanza universitaria. En V. Álvarez Rojo, y A. Lázaro Martínez (Coords.), Calidad de las universidades y orientación educativa (pp. 215-247). Málaga: Aljibe.

Ames, C. (1992). Classrooms: Goals, structures and student motivation. Journal of Educational Psychology, 84, 261-271.

Bamburg, J. (2004) Raising expectations to improve student learning. En: http://www.ncrel.org/sdrs/areas/issues/educatrs/leadrshp/le0bam.htm

Brophy, J. (2004). Motivating students to learn.Mahwah, New Jersey: LEA.

Caprara, G. V., Barbaranelli, C., Borgogni, L. y Steca, P. (2003). Efficacy beliefs as determinants of teachers' job satisfaction. Journal of Educational Psychology, 95, 821-832.

Caprara, G. V., Barbaranelli, C., Borgogni, L., Petitta, L. y Rubinacci, A. (2003). Teachers', school staff's and parents' efficacy beliefs as determinants of attitude toward school. European Journal of Psychology of Education, 18, 15-31.

Catena, A., Ramos, M.M. y Trujillo, H.M. (2003). Análisis multivariado. Un manual para investigadores. Madrid: Biblioteca Nueva.

Cousins, J. y Walker, C. (1995a). Personal teacher efficacy as a predictor of teachers' attitudes toward applied educational research. Comunicación presentada al Annual Meeting of the Canadian Association for the Study of Educational Administration, Montreal.

Cousins, J. y Walker, C. (1995b). Predictors of educators' valuing of systemic inquiry in schools. Canadian Journal of Program Evaluation, Special Issue, 25-35.

Dweck, C. S., \& Leggett, E. L. (1988). A social-cognitive approach to motivation and personality. Psychology Review, 95, 256-273.

Elliot, A. J., \& McGregor, H. A. (2001). A 2x2 achievement goal framework. Journal of Personality and Social Psychology, 80, 501-519.

Gibson, S. y Dembo, M. (1984). Teacher efficacy: a construct validation. Journal of Educational Psychology, 76, 569-582.

González, J. y Wagenaar, R. (2003). Tuning educational structures in Europe. Informe Final - Proyecto Piloto, Fase Uno. Bilbao: Universidad de Deusto.

Guskey, T. (1988). Teacher efficacy, self-concept, and attitudes toward the implementation of instructional innovation. Teaching and Teacher Education, 4, 63-69.

Hoy, W.K. y Woolfolk, A. (1990). Socialization of student teachers. American Educational Research Journal, 27, 279-300.

Klassen, R.M., Bong, M., Usher, E.L., Chong, W.H., Huan, V.S., Wong, I.Y.F. y Georgiou, T. (2009). Exploring the validity of a teachers' self-efficacy scale in five countries. Contemporary Educational Psychology, 34, 67-76.

Pintrich, P.R. (2000). Multiple goals, multiple pathways: The role of goal orientation in learning and achievement.
Journal of Educational Psychology, 92, 544-555.

Pintrich, P. R. (2003). A motivational science perspective on the role of student motivation in learning and teaching contexts. Journal of Educational Psychology, 95, 667-686.

Rodríguez, G. (2002). El reto de enseñar hoy en la Universidad. En V. Álvarez y A. Lázaro (Coods.), Calidad de las universidades y orientación universitaria (pp. 49-58). Málaga: Aljibe.

Skaalvik, E.M. y Skaalvik, S. (2007). Dimensions of teacher self-efficacy and relations with strain factors, perceived collective teacher efficacy, and teacher burnout. Journal of Educational Psychology, 99, 611-625.

Stein, M. K. y Wang, M. C. (1988). Teacher development and school improvement: The process of teacher change. Teaching and Teacher Education, 4, 171-187.

Tschannen-Moran M. y Woolfolk, A. (2001). Teacher efficacy: capturing an elusive construct. Teaching and Teacher Education. 17, 783-805.

Valle, A., Cabanach, R.G., Núñez, J.C., González-Pienda, J.A., Rodríguez, S. y Piñeiro, I. (2003). Multiple goals, motivation and academic learning. British Journal of Educational Psychology, 73, 71-87.

Valle, A., Núñez, J. C., Cabanach, R. G., Rodríguez, S., González-Pienda, J. A. y Rosário, P. (2009). Perfiles motivacionales en estudiantes de Secundaria: Análisis diferencial en estrategias cognitivas, estrategias de autorregulación y rendimiento académico. Revista Mexicana de Psicología, 26, 113-124.

Valle, A., Núñez, J. C., Rodríguez, S., Cabanach, R. G., González- Pienda, J. A. y Rosário, P. (en prensa). Perfiles Motivacionales y Diferencias en Variables Afectivas, Motivacionales y de Logro. Universitas Psychologica.

Wolters, C.A. y Daugherty, S.G. (2007). Goals structures and teachers' sense of efficacy: Their relation and association to teaching experience and academic level. Journal of Educational Psychology, 99, 181-193.

Woolfolk, A. (2004). What do teachers need to know about self-efficacy? Comunicación presentada al Annual Meeting of the American Educational Research Association, San Diego.

Zimmerman, B. J. (2000). Attaining self-regulation. A social cognitive perspective. In M. Boekaerts, P. Pintrich y M. Zeidner (Eds.), Handbook of self-regulation. New York (pp. 13-39). San Diego: Academic press.

Zimmerman, B. J. (1998). Developing self-fulfilling cycles of academic regulation: An analysis of exemplary instructional models. In D. H. Schunk \& B. J. Zimmerman (Eds.), Self-Regulated learning. From teaching to Self-Reflective Practice (pp. 1-19). Hillsdale, NJ: Lawrence Erlbaum Associates, Inc.

Fecha de recepción: 29 de junio de 2009 Fecha de aceptación: 5 de noviembre de 2009 